\section{Carbohydrate Accumulation and Flowering-related Gene Expression Levels at Different Developmental Stages of Terminal Shoots in Litchi chinensis}

\author{
Hai-Fang Yang \\ College of Horticulture, South China Agricultural University, Guangzhou \\ 510642, China
}

Hye-Ji Kim

Department of Horticulture and Landscape Architecture, Purdue University, West Lafayette, IN 47907-201

Hou-Bin Chen, MD

College of Horticulture, South China Agricultural University, Guangzhou 510642, China

\section{Jillur Rahman}

Horticulture Research Centre, Bangladesh Agricultural Research Institute, Joydebpur, Gazipur-1701, Bangladesh

\section{Xing-Yu Lu and Bi-Yan Zhou ${ }^{1}$ \\ College of Horticulture, South China Agricultural University, Guangzhou 510642, China}

Additional index words. litchi, flowering, carbohydrate, flowering-related gene

\begin{abstract}
Litchi trees flower at the apex of terminal shoots. Flowering is affected by the maturity of terminal shoots before growth cessation occurs during the winter. In this study, we focused on changes of flowering in three important cultivars, Guiwei, Feizixiao, and Huaizhi, from Dec. 2012 to Mar. 2013 under natural winter conditions. Flowering rate, carbohydrate accumulation, and expression of the flowering-related genes were determined at three different developmental stages of terminal shoots with dark green, yellowish green and yellowish red leaves, respectively. The results showed that the total soluble sugar and starch contents in the dark green leaves were the highest, whereas those in the yellowish red leaves were the lowest. Trees with dark green terminal shoots had the highest flowering rates, whereas those with yellowish green or yellowish red shoots had relatively lower flowering rates. SPAD was highest in dark green leaves and lowest in yellowish red leaves at the start of the trial. The SPAD value of yellowish red leaves slightly increased but did not reach the levels of the dark green leaves, whereas levels of the other leaf stages remained fairly constant. Expression level of the litchi homolog FLOWERING LOCUS C (LCFLC), the floral inhibitor in yellowish red leaves, increased from 16 Jan., whereas that in dark green leaves declined to a level lower than the yellowish red leaves on 4 Feb. Expression level of the litchi homolog CONSTANTS $(\mathrm{LcCO})$, the floral promoter in dark green leaves, was higher than that of yellowish red leaves before 26 Jan. Expression level of the litchi homolog FLOWERING LOCUS T 2 (LcFT2), encoding florigen, was higher in dark green leaves than in the other two leaf types. Our results suggest that terminal shoots should be matured and leaves should turn green for successful flowering. Mature leaves had higher expression levels of the floral promoter and florigen. In litchi production, leaves of the terminal shoots (potential flowering branches) should be dark green during floral induction and differentiation stages, and winter flushes should be removed or killed.
\end{abstract}

Received for publication 29 July 2014. Accepted for publication 25 Aug. 2014

This study was funded by the National Natural Science Foundation (31071760) and the Agricultural Industry Project (CARS-33-08) by the Ministry of Agriculture.

${ }^{1}$ To whom reprint requests should be addressed; e-mail zhoubiyan@scau.edu.cn.
Litchi (Litchi chinensis Sonn.) is an evergreen fruit tree widely cultivated in southeast Asia. However, unreliable flowering is a serious problem in the litchi industry. Litchi flowering is induced by low temperatures and enhanced by drought in winter (Chaikiattiyos et al., 1994; Chen and Huang, 2005; Menzel and Simpson, 1988). Although temperature and soil moisture are low enough during winter to induce flowering, maturity of terminal shoots must occur before growth cessation in winter to avoid poor flower set.

Normally litchi trees flower at the apex of terminal shoots. The accumulation of carbohydrates in these shoots is dependent on mature leaves, which have photosynthetic capacity. The carbon-to-nitrogen in plants has been considered as a factor affecting flowering. Evidence has revealed an intricate and integrated nitrogen and carbon sensing and signaling network to control various developmental processes (Coruzzi and Zhou, 2001; Gibson, 2005). Litchi flowering is considered to be related to the patterns of carbohydrate distribution in trees (Chen et al., 2004). Carbohydrates are accumulated in leaves and branches of litchi trees at fall for flowering (Menzel et al., 1995).

In Arabidopsis, CONSTANS (CO) codes a zinc finger transcription factor, which activates the expression of FLOWERING LOCUS T (FT) by binding to FT regulatory regions (An et al., 2004). The FT protein is a component of the mobile flowering signal "florigen" that moves on its expression in the vascular tissue of leaves to the shoot apex (Yang et al., 2007). FLC (FLOWERING LOCUS C) is a floral repressor. Vernalization decreases $F L C$ expression, which allows the induction of $F T$ in leaves and the transition from vegetative to reproductive growth (Wellmer and Riechmann, 2010).

In the present study, we focused on three important commercially cultivated cultivars, Guiwei, Feizixiao, and Huaizhi. We investigated the carbohydrate accumulation at different developmental stages of terminal shoots in relation to flowering. We also determined expression levels of the litchi homolog $C O, F T$, and $F L C$ in these trees to determine an appropriate developmental stage for litchi flowering.

\section{Materials and Methods}

Plant material and experiment procedures. The experiment was carried out from Dec. 2012 to Mar. 2013. Four-year-old potted litchi tree cvs. Guiwei, Feizixiao, and Huaizhi from the experimental orchard of the South China Agricultural University, Guanzhou, China, were propagated by air layering. Five replicate trees whose leaves of the terminal shoots were dark green, yellowish green, or yellowish red were selected for each cultivar, and each consisted of 15 to 20 terminal shoots. The trees were subjected to chilling temperatures under natural winter conditions as shown in Figure 1. Morphology of the dark green shoots at the induction stage (before panicle primordia emerged) and floral differentiation stage (after panicle primordia emerged) is shown in Figures $2 \mathrm{~A}-\mathrm{B}, 2 \mathrm{G}-\mathrm{H}$, and $2 \mathrm{M}-\mathrm{N}$ and that of the yellowish green and yellowish red shoots at the same time is shown in Figs. 2C-F, 2I-L, and 2O-R. Panicle primordia of 'Guiwei' and 'Huaizhi' were visible in late January of 2013, and those of 'Feizixiao' were visible in midJanuary of that year. Flowering rate was calculated from five tested trees and the percentage of 


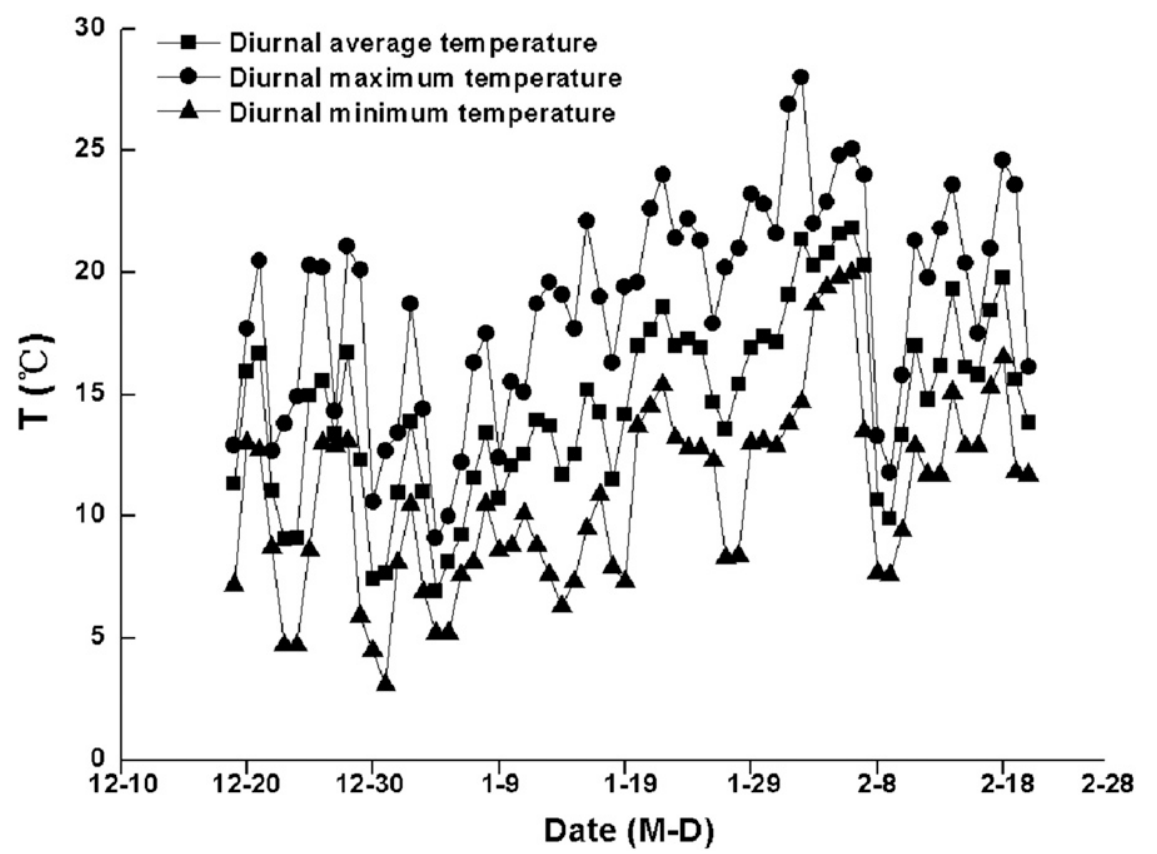

Fig. 1. Temperature changes in the winters of 2012-13 showing diurnal maximum temperature, diurnal average temperature, and diurnal minimum temperature in the experimental orchard of the South China Agricultural University. M-D = month-date. immediately frozen in liquid nitrogen for determination of soluble sugar, starch, and gene expression levels.

Determination of soluble sugar and starch. Samples were ground to powder and distilled water was added. Samples were incubated in a water bath at $90{ }^{\circ} \mathrm{C}$ for $30 \mathrm{~min}$ and were centrifuged at $8000 \mathrm{rpm}$ for $15 \mathrm{~min}$. The supernatant was used to determine soluble sugar, sucrose, and fructose according to the method of Xue (1985) and glucose according to the method of Zhou (2004). Extraction of starch and determination are according to the method of Xu et al. (1998).

Analysis of real-time quantitative reverse transcription-polymerase chain reaction. Total RNA was extracted from leaves of terminal shoots using an RNA extraction kit (Hua Yue Yang, China). First-strand cDNA was generated and primers for quantitative polymerase chain reaction (qPCR) were designed and synthesized by Sangon Biotech (Shanghai) Co., Ltd. (Shanghai, China). All the primers are shown in Table 1. qPCR was performed on a Bio-Rad iQ5 Optical System Real Time PCR System (Bio-Rad, USA) according to the method of Liu et al.
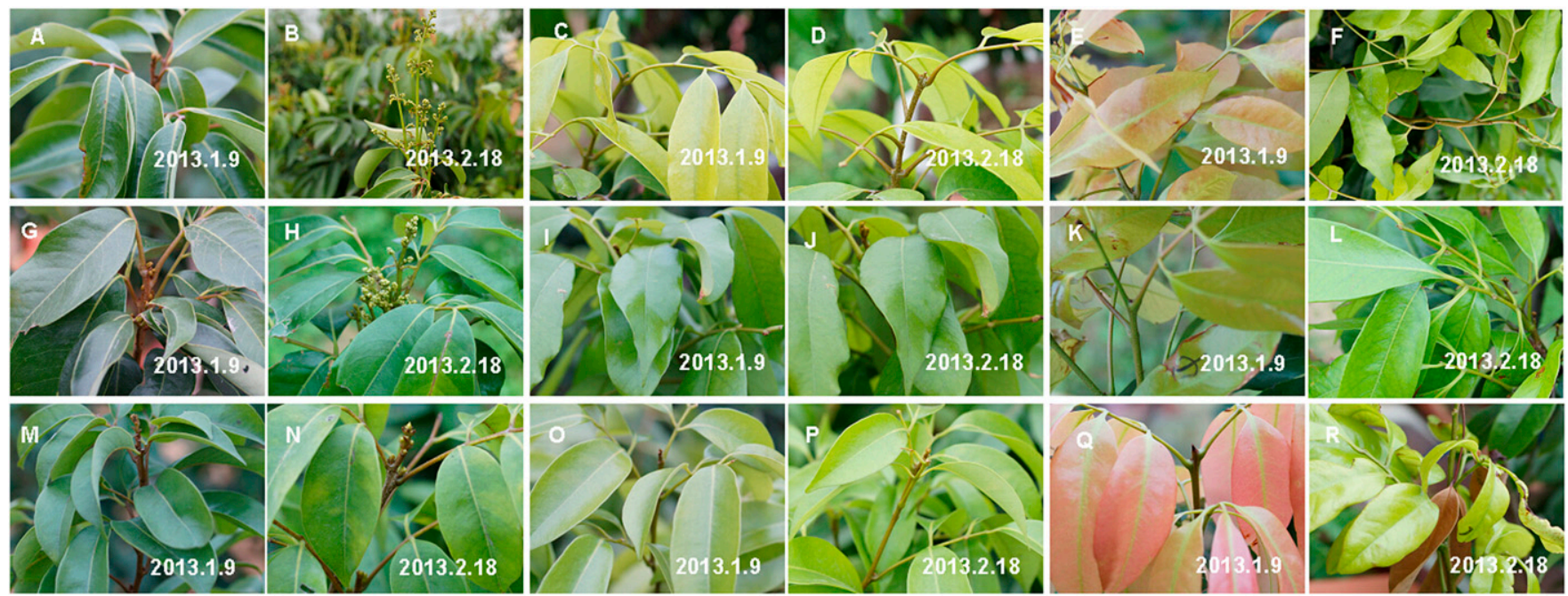

Fig. 2. Dark green leaves of 'Guiwei' (A-B), 'Feizixiao' (G-H), and 'Huaizhi' (M-N); yellowish green leaves of 'Guiwei' (C-D), 'Feizixiao' (I-J), and

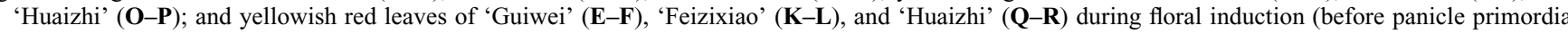
emerged) and differentiation (after panicle primordia emerged) stages. Panicle primordia of 'Guiwei' and 'Huaizhi' were visible in late January of 2013, and those of 'Feizixiao' were visible in mid-January. Photos taken on 9 Jan. 2013 indicate trees were in floral induction stage, and those on 18 Feb. 2013 were in the floral differentiation stage.

flowering terminal shoots was calculated from the flowering trees after anthesis (late March). Six to 10 panicles in one flowering tree were randomly selected and enclosed in nylon nets before flowering to collect all the abscised flowers. Numbers of flowers including male and female flowers per panicle were determined after anthesis. After panicle primordia emerged, phloem and xylem of the terminal shoots from three replicate trees were collected and immediately frozen in liquid nitrogen. The SPAD values correspond to the amount of chlorophyll in leaves (Wang et al., 2004; Yamamoto et al., 2002). SPAD values in leaves of the terminal

Table 1. Primer sequences of the genes for quantitative reverse transcription-polymerase chain reaction.

\begin{tabular}{lll}
\hline Homology gene & \multicolumn{1}{c}{$\begin{array}{c}\text { Forward } \\
\text { primer }\left(5^{\prime} \rightarrow 3^{\prime}\right)\end{array}$} & \multicolumn{1}{c}{$\begin{array}{c}\text { Reverse } \\
\text { primer }\left(5^{\prime} \rightarrow 3^{\prime}\right)\end{array}$} \\
\hline Actin & AGTTTGGTTGATGTGGGAGAC & TGGCTGAACCCGAGATGAT \\
$L c F L C$ & AAAATAGCACATCCAACAGGC & AACAAACTTACATAGGTAGTGAGGC \\
$L c C O$ & GACTCAGCCGACTCGATTGTC & GTTATCGGCCGGCAACAG \\
$L c F T 2$ & CCAAGTGAACCAAGCCTGAG & GGAACAACACGAATACGAACC \\
\hline
\end{tabular}

shoots from five replicate trees were measured using a chlorophyll meter (SPAD, Model 502; Minolta Co., Japan). On 26 Dec. 2012, 5 Jan., 16 Jan., 26 Jan., and 4 Feb. 2013, leaves from three replicate trees were sampled and
(2013). The qPCRs were run as follows: $50{ }^{\circ} \mathrm{C}$ for $2 \mathrm{~min}, 95^{\circ} \mathrm{C}$ for $10 \mathrm{~min}$, followed by 40 cycles of $95^{\circ} \mathrm{C}$ for $30 \mathrm{~s}, 60^{\circ} \mathrm{C}$ for $30 \mathrm{~s}$, and $72{ }^{\circ} \mathrm{C}$ for $30 \mathrm{~s}$ in 96 -well optical reaction plates (Bio-Rad, USA). Each qRT-PCR 
Table 2. Effects of developmental stage of terminal shoot on flowering of 'Guiwei', 'Feizixiao', and 'Huaizhi' litchi.

\begin{tabular}{|c|c|c|c|c|c|c|}
\hline Cultivars & $\begin{array}{c}\text { Developmental stage } \\
\text { of leaves }\end{array}$ & $\begin{array}{c}\text { Percentage of } \\
\text { flowering plants }(\%)\end{array}$ & $\begin{array}{l}\text { Percentage of flowering } \\
\text { terminal shoots }(\%)\end{array}$ & $\begin{array}{l}\text { Number of flowers } \\
\text { per panicle }\end{array}$ & $\begin{array}{c}\text { Percentage of } \\
\text { female flowers }(\%)\end{array}$ & $\begin{array}{c}\text { Percentage of } \\
\text { male flowers }(\%)\end{array}$ \\
\hline \multirow{2}{*}{ Guiwei } & Yellowish green & 0 & $0 \pm 0.0$ & $0 \pm 0.0$ & $0 \pm 0.0$ & $0 \pm 0.0$ \\
\hline & Yellowish red & 0 & $0 \pm 0.0$ & $0 \pm 0.0$ & $0 \pm 0.0$ & $0 \pm 0.0$ \\
\hline \multirow{2}{*}{ Feizixiao } & Yellowish green & 20 & - & - & - & - \\
\hline & Yellowish red & 60 & $37.3 \pm 14.9$ & $525.4 \pm 265.0$ & $9.9 \pm 3.7$ & $90.1 \pm 3.7$ \\
\hline Huaizhi & Dark green & 100 & $76.0 \pm 6.3$ & $309.5 \pm 45.4$ & $8.2 \pm 1.6$ & $91.8 \pm 1.6$ \\
\hline
\end{tabular}

Values are means \pm SE. “_” = data not shown.

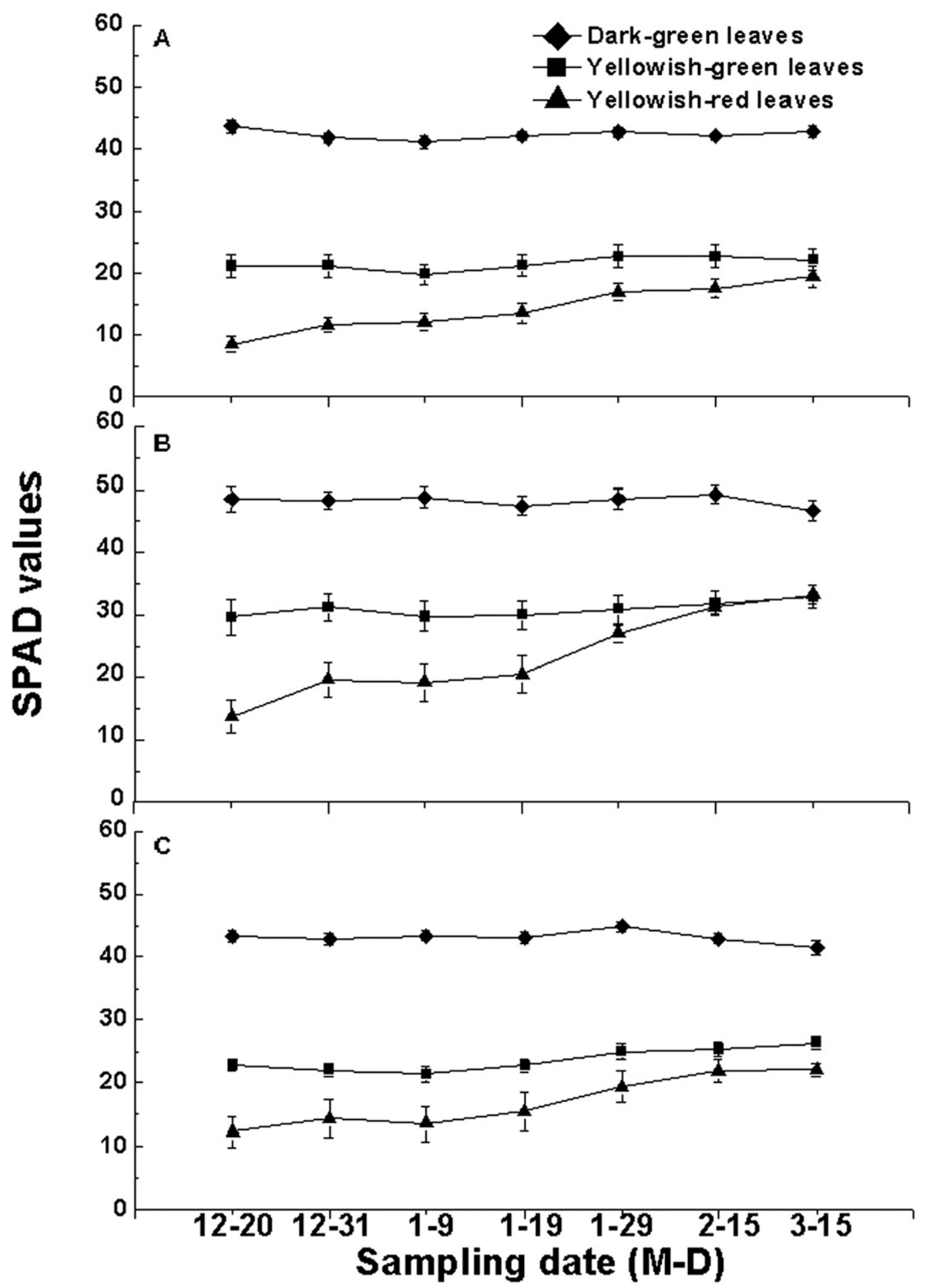

Fig. 3. Changes in SPAD values of the leaves in 'Guiwei' (A), 'Feizixiao' (B), and 'Huaizhi' (C) litchi trees at different developmental stages during floral induction and differentiation stages. Values represent means of five replicates and bars indicate sES. M-D = month-date. 


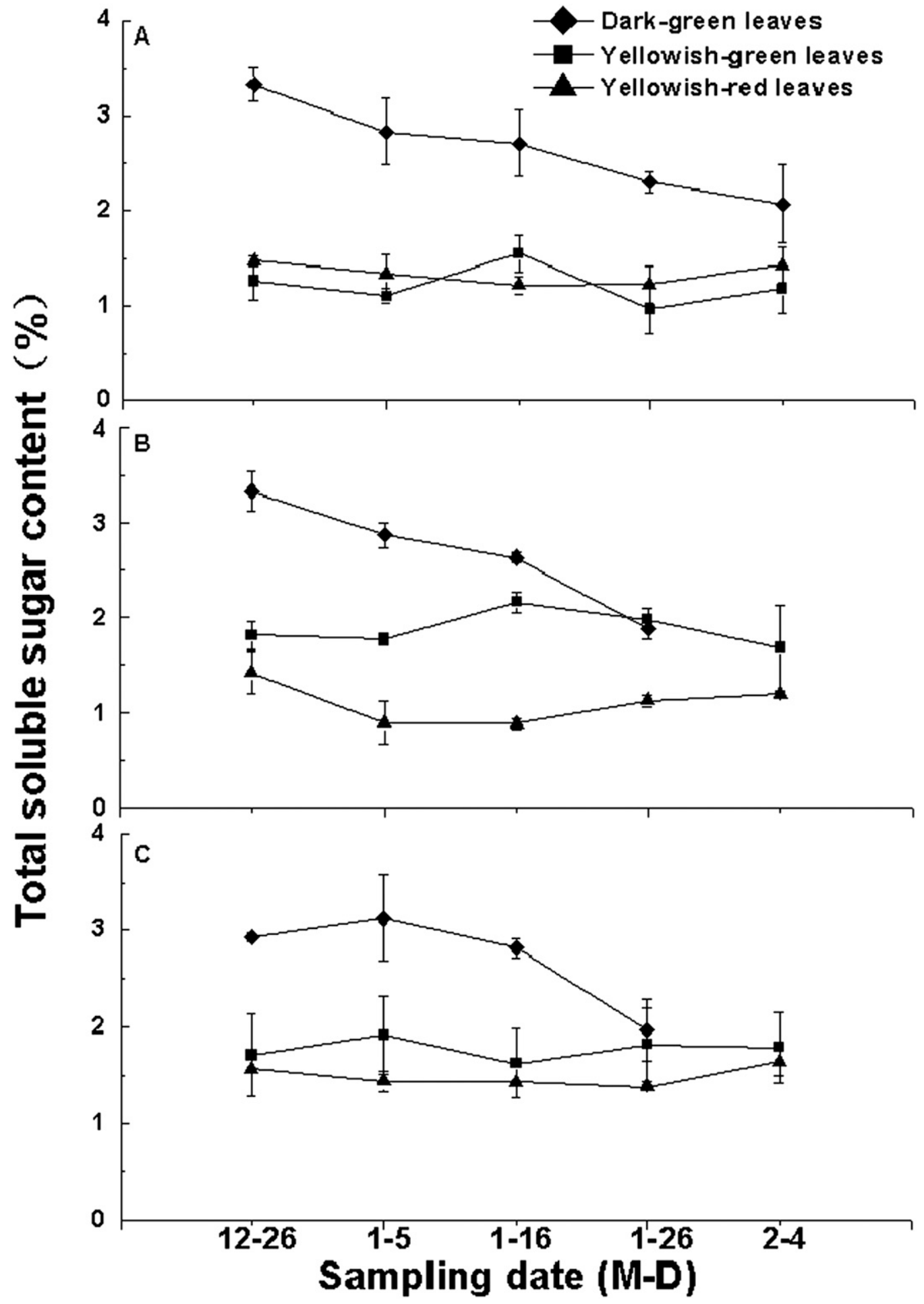

Fig. 4. Changes in total soluble sugar contents of the leaves of 'Guiwei' (A), 'Feizixiao' (B), and 'Huaizhi' (C) litchi trees at different developmental stages during floral induction and differentiation stages. Values represent means of three replicates and bars indicate SES. M-D = month-date.

analysis was performed in triplicate. Expression levels of the tested reference genes were determined by $\mathrm{Ct}$ values and calculated by $2^{-\triangle \triangle \mathrm{Ct}}$ (Livak and Schmittgen, 2001).

Statistical analysis. Data were subjected to analysis of variances using a SPSS 19.0 program (SPSS Inc., Chicago, IL). The differences among treatment means were evaluated by Duncan's multiple range test at a $0.05 P$ level. SE was calculated by Microsoft Excel 2010 (Microsoft Co., Redmond, WA).

\section{Results}

Effects of developmental stages on flowering of litchi trees. Percentage of flowering was higher in terminal shoots with dark green leaves than that of the yellowish green or yellowish red leaves in 'Guiwei', 'Feizixiao', and 'Huaizhi' trees. The percentage of flowering of terminal shoots was more than $60 \%$ in all the flowering trees with dark green terminal flushes. Among those, 'Feizixiao' developed the largest number of flowers in one panicle. In 'Guiwei' litchi trees, no flowers were found in the yellowish green or yellowish red shoots. Among the flowering branches, the dark green shoot of 'Guiwei' produced the highest percentage of female flowers (Table 2).

Changes in SPAD values of terminal shoot at different developmental stages. SPAD values of dark green leaves of the cultivars were $\approx 45$ to 50 at the start of the 


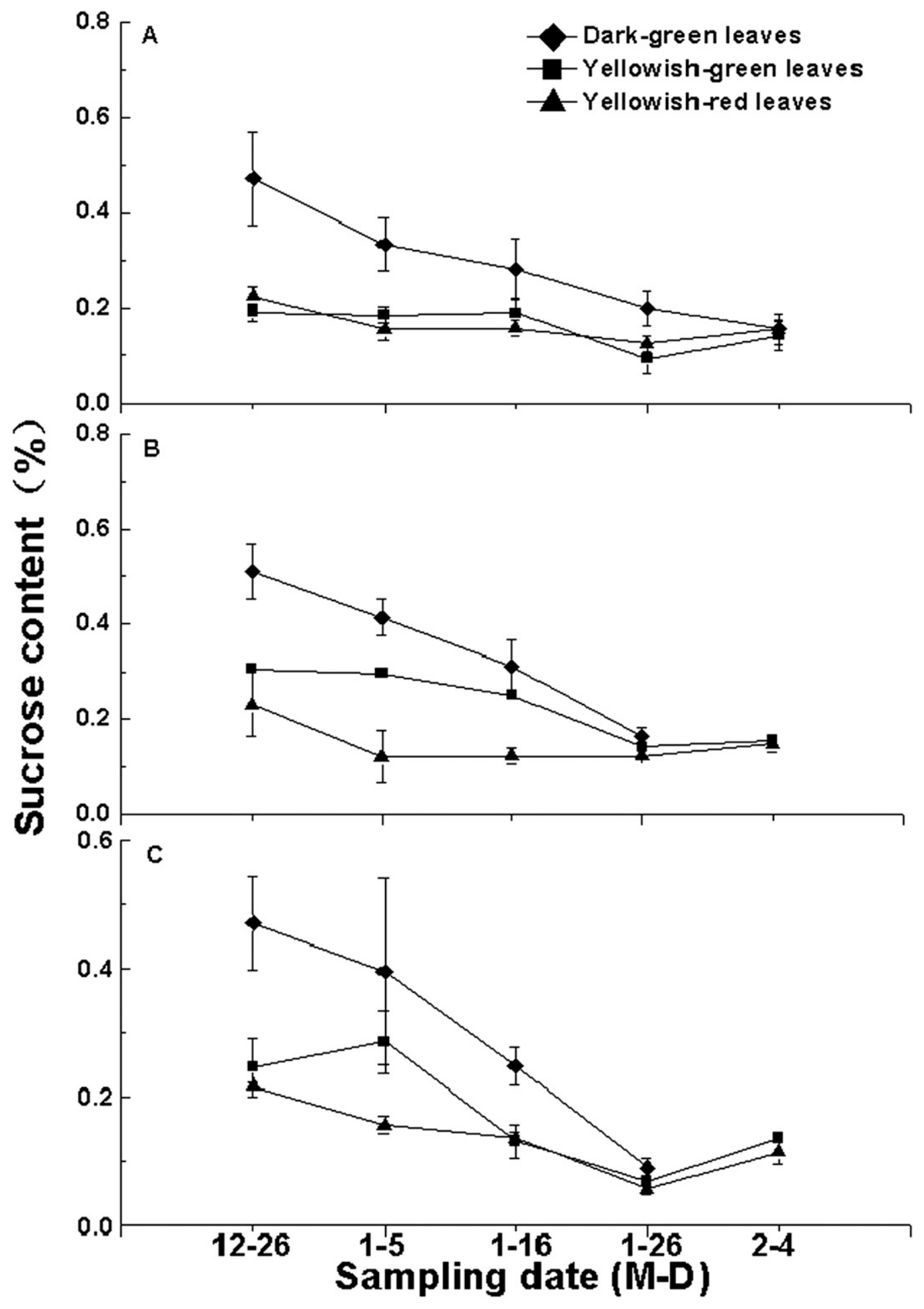

Fig. 5. Changes in sucrose contents of the leaves of 'Guiwei' (A), 'Feizixiao' (B), and 'Huaizhi' (C) litchi trees at different developmental stages during floral induction and differentiation stages. Values represent means of three replicates and bars indicate sES. M-D $=$ month-date.

trial, whereas those of yellowish green and yellowish red leaves were 20 to 30 and 10 to 15 , respectively. SPAD values of dark green leaves or yellowish green leaves of the three cultivars were found to be relatively stable during winter. SPAD values of yellowish red leaves, however, slightly increased during the floral induction and differentiation stages (Fig. 3).

Changes in sugar and starch contents in terminal shoots at different developmental stages. Total soluble sugar contents of the dark green leaves in three cultivars decreased from 26 Dec. 2012 to 4 Feb. 2013, whereas those in the yellowish green and yellowish red leaves remained at lower levels and were relatively stable during the period. On the whole, those levels in the dark green leaves were higher than in the yellowish green or yellowish red leaves (Fig. 4).

Sucrose contents of the dark green leaves of all three cultivars decreased from 26 Dec.
2012 to 4 Feb. 2013, showing similar trends as those of total soluble sugar contents. Sucrose contents in dark green leaves were the highest, whereas those in the yellowish green and yellowish red leaves were relatively low with slight differences among varieties (Fig. 5). Changes in the glucose contents, however, showed different profiles from those of the total soluble sugar contents and sucrose contents. Only those of the dark green leaves in 'Guiwei' and 'Feizixiao' 


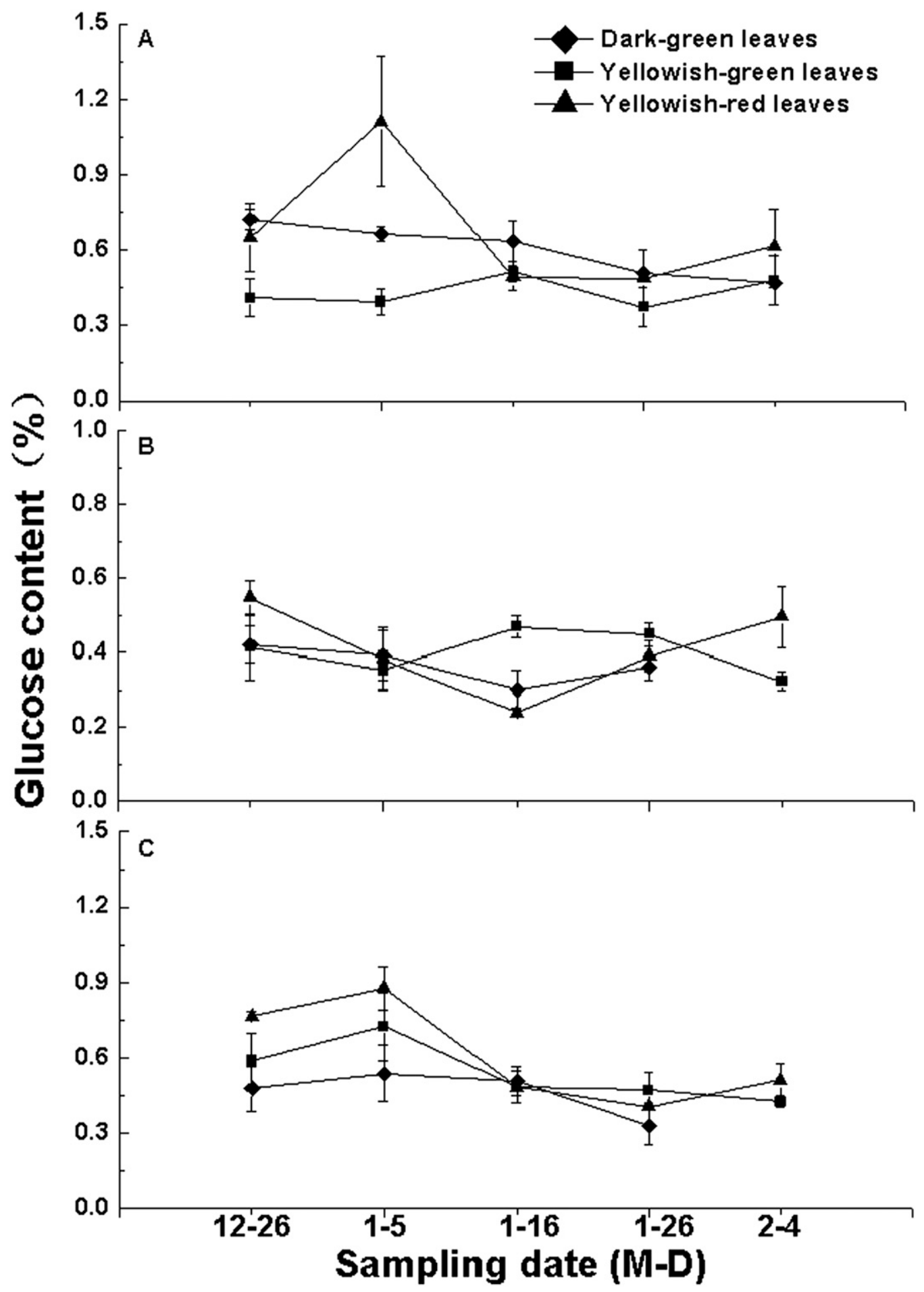

Fig. 6. Changes in glucose contents of the leaves of 'Guiwei' (A), 'Feizixiao' (B), and 'Huaizhi' (C) litchi trees at different developmental stages during floral induction and differentiation stages. Values represent means of three replicates and bars indicate sEs. M-D $=$ month-date.

showed slightly decreasing trends during the floral induction and differentiation stages (Fig. 6). In general, fructose contents of the leaves at three developmental stages decreased during the trial period in all cultivars, showing similar trends as those of sucrose (Figs. 5 and 7).

Starch contents of the dark green leaves in 'Guiwei', 'Feizixiao', and 'Huaizhi' were higher than those of the yellowish green and yellowish red leaves. In sum, starch contents of the cultivars were relatively stable during the floral induction and differentiation stages (Fig. 8).

Sugar and starch contents in xylem and phloem of terminal shoots at different developmental stages. To compare the sugar and starch contents of terminal branches at different developmental stages, xylem and phloem tissues were collected separately from branches after panicle primordia emerged. As shown in Figure 9, the contents of total soluble sugar, sucrose, glucose, fructose, and starch in xylem of 'Guiwei' in three stages of leaves were not found to be significantly different. However, in 'Huaizhi', the xylem of the yellowish green branches contained relatively higher levels of total soluble sugar, sucrose, fructose, and starch. In 'Feizixiao', only sucrose and fructose levels of the dark green leaves were lower than those of yellowish green and yellowish red leaves.

In the phloem of 'Guiwei' litchi trees, the contents of total soluble sugar, sucrose, fructose, and starch in the dark green branches were higher than those in yellowish 


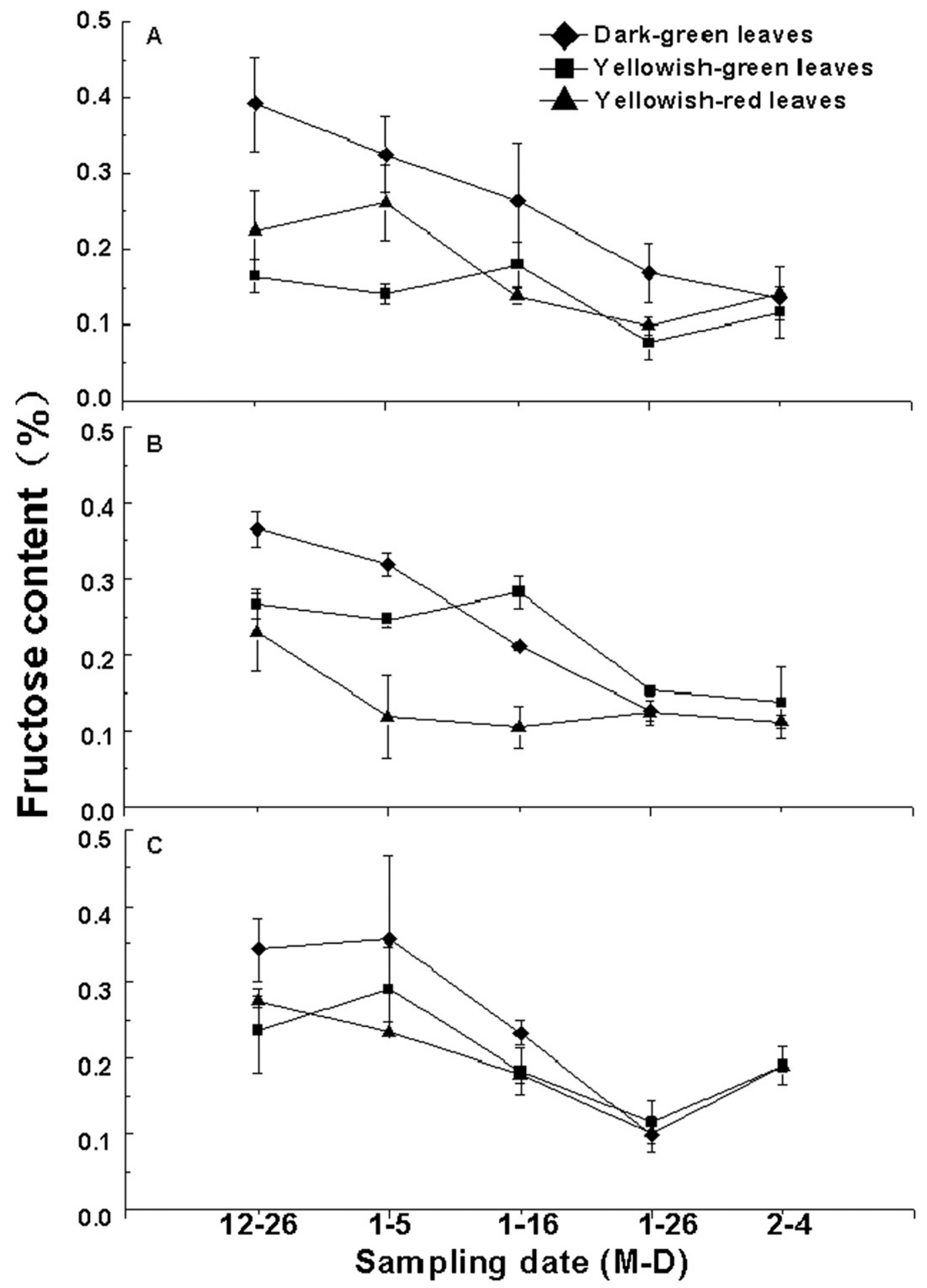

Fig. 7. Changes in fructose contents of the leaves of 'Guiwei' (A), 'Feizixiao' (B), and 'Huaizhi' $(\mathbf{C})$ litchi trees at different developmental stages during floral induction and differentiation stages. Values represent means of three replicates and bars indicate sEs. M-D = month-date.

green or yellowish red branches, whereas the contents of glucose in the three different types of branches were the same. In the other two cultivars, no significant differences were found among the three types of branches (Fig. 9).

LcFLC, LcCO, and LcFT2 expressions in the leaves of different developmental stages. To investigate the flowering-related gene expression in the leaves of terminal shoots at different developmental stages, the leaves of 'Huaizhi' potted trees were sampled for determination of gene expression. LcFLC expression of the yellowish green and yellowish red branches increased to the highest levels on 5 Jan. and then decreased, whereas that of the dark green branches remained relatively stable and decreased to a low level on 4 Feb. (Fig. 10A).

$L c C O$ expression of the dark green and yellowish green leaves increased to the highest levels on 5 Jan. and decreased afterward, whereas that of the yellowish red leaves was at a low level on 26 Dec. and gradually increased (Fig. 10B).

LcFT2 expression of the dark green leaves increased from 26 Dec. to 26 Jan., whereas that of the yellowish green or yellowish red leaves remained at a low level during the entire period. The expression level of the dark green leaves was much higher than the other types of leaves. For example, on 16 Jan., its related expression level was 


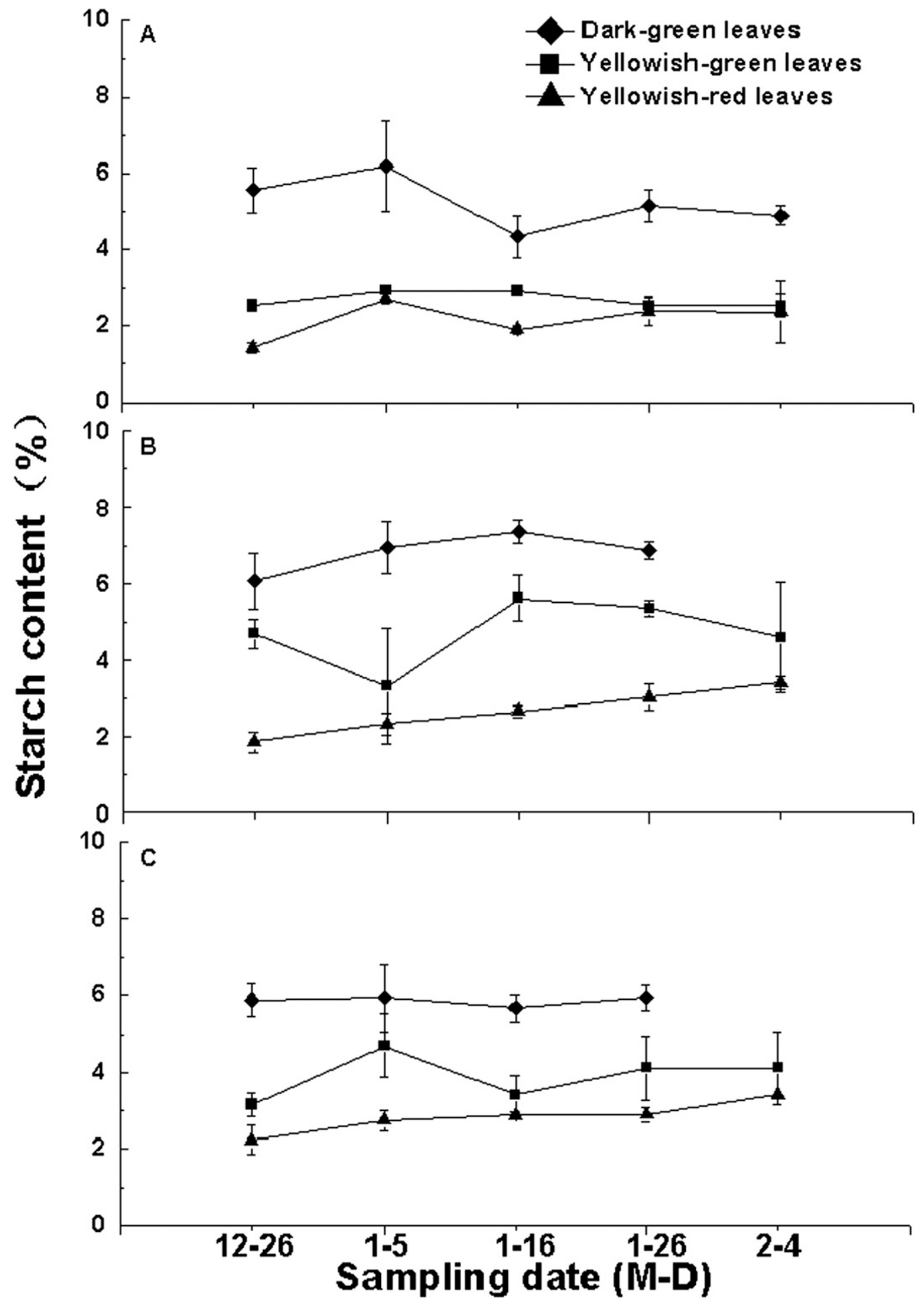

Fig. 8. Changes in starch contents of the leaves of 'Guiwei' (A), 'Feizixiao' (B), and 'Huaizhi' (C) litchi trees at different developmental stages during floral induction and differentiation stages. Values represent means of three replicates and bars indicate sEs. M-D $=$ month-date.

46.7-fold and 290.3-fold higher than those of the yellowish green and yellowish red leaves, respectively (Fig. 10C).

\section{Discussion}

Carbohydrates include soluble sugar and starch. Normally, plants contain sucrose, glucose, and fructose as soluble sugars. It has been emphasized that carbohydrates play an important role in flowering (Chen et al., 2004; Menzel et al., 1995). Sugars have conventionally been viewed as resources for respiration and metabolic intermediates as well as structural components (Sheen et al., 1999). Floral formation is promoted by ringing, root pruning, and fruit thinning, which could restrict vegetative growth, resulting in carbohydrate accumulation. Decrease in floral formation has been reported to be related to the duration and intensity of shading, which could decrease the carbohydrate accumulation level (Davenport and Stern, 2005; Ito et al., 2002). Furthermore, sugars are believed to be signaling molecules that control gene expression and developmental processes in plants (Jang and Sheen, 1997; Sheen et al., 1999). In this study, we investigated the relationship between carbohydrate accumulation levels and flowering formation. We 

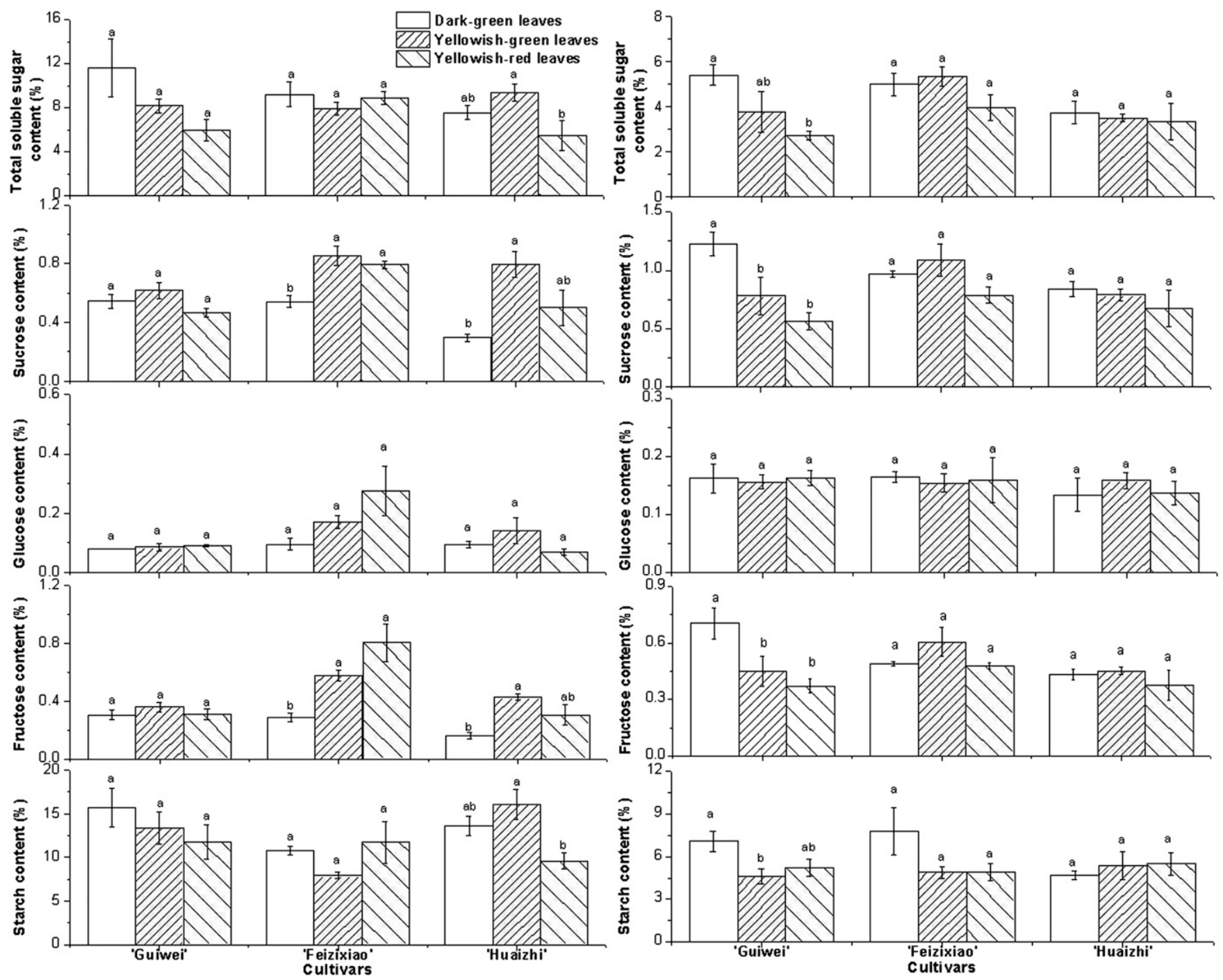

Fig. 9. Sugars and starch contents in xylem (left) and phloem (right) of the terminal shoots at different developmental stages after panicle primordia emerged. Values represent means of three replicates and bars indicate sEs. Different letters indicate significant difference at $P=0.05$ among the developmental stages within a given cultivar according to Duncan's multiple range test.

selected three types of terminal shoots with dark green, yellowish green, and yellowish red leaves. The results showed that the leaves in these three types of branches contained different levels of carbohydrates; the total soluble sugar and starch contents in the dark green leaves were the highest, whereas those in the yellowish red leaves were the lowest. In accordance with carbohydrate accumulation, trees with dark green terminal shoots had the highest flowering rate, whereas those with yellowish green or yellowish red shoots had relatively lower flowering rates, suggesting that terminal shoots should mature and leaves should turn dark green for successful flowering. In practice, whether the terminal shoots mature before floral induction is critical for litchi production. Immature shoots in winter (winter flushes) should be removed or killed by spaying with ethephon or paraquat (Zhou et al., 2012, 2014).

To investigate whether the leaves of the terminal shoots had any change during the floral induction and differentiation stages, we determined SPAD values that correspond to the amount of chlorophyll (Wang et al., 2004; Yamamoto et al., 2002). We found that SPAD values of the yellowish red leaves slightly increased but did not reach to those of the dark green leaves over the trial, further confirming that leaves in the terminal shoots should turn dark green before the floral induction stage. SPAD values of the dark green leaves gradually decreased in 'Guiwei', 'Feizixiao', and 'Huaizhi', indicating that the color of the dark green leaves became lighter. Yin et al. (2008) also found that the dark green leaves in 'Nuomici' turned light green and the concentration of chlorophylls decreased with the transition from vegetative growth to reproductive growth. They suggested that this change was attributed to low-temperature stress and increase in starch accumulation. In our previous study in which litchi trees were subjected to low temperature and water stress in winter, we found that hydrogen peroxide as reactive oxygen species accumulated and electrolyte leakage in- creased in winter (Zhou et al., 2010a) Exogenous application of the hydrogen peroxide inducer methyl viologen dichloride hydrate promoted flowering (Zhou et al., 2012); it was suggested that the decrease in chlorophyll content might be a result of stress or hydrogen peroxide accumulation. Growers regard this phenomenon as a cue for perfect flowering. However, as to starch, our present results showed that starch content in the dark green leaves did not increase during the floral induction and differentiation period (Fig. 8). Further investigation should be carried out to elucidate the role of starch in litchi flowering.

Floral induction and differentiation are controlled by environmental conditions and developmental regulation. The complexity of this regulation is created by an intricate network of signaling pathways comprising many flowering-related genes. In the present study, we determined $L c C O, L c F L C$, and $L c F T 2$ expression to elucidate whether the developmental stage of the terminal shoots affected those flowering-related genes. In 


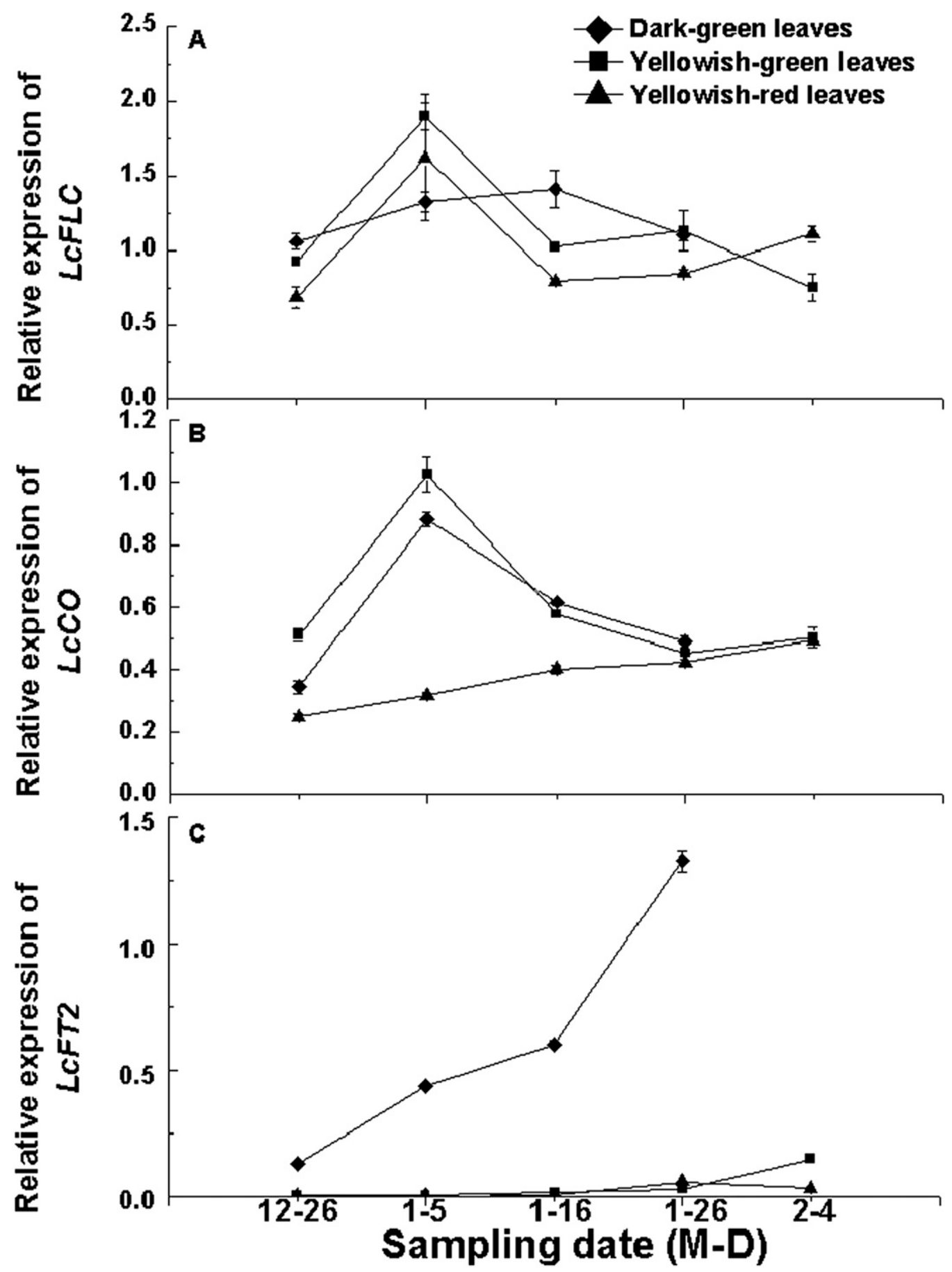

Fig. 10. Relative expression of $L c F L C(\mathbf{A}), L c C O(\mathbf{B})$, and $L c F T 2(\mathbf{C})$ during floral induction and differentiation stages of 'Huaizhi'. Values represent means of three replicates and bars indicate SES. M-D = month-date.

Arabidopsis, $\mathrm{CO}$ acts as a floral promoter and promotes flowering in long-day conditions (Samach et al., 2000). However, constitutive overexpression of $C O$ in plants causes rapid flowering even in short days, demonstrating that $C O$ can trigger flowering. $C O$ appears to promote flowering by directly up-regulating the expression of the $F T$ in noninductive conditions (Hayama and Coupland, 2003). FT protein as a florigen and a mobile signal migrated from leaves to the apical meristem to promote floral initiation in several plant species (Yang et al., 2007), activating the downstream flowering-related genes ( $\mathrm{Li}$ et al.,
2010) such as LEAFY (LFY), APETALAI (AP1), APETALA3 (AP3), AG (Mouradov et al., 2002; Wagner et al., 1999; Zhou et al., 2010b). Our results showed that $L c C O$ expression of the dark green leaves was higher than that of the yellowish red leaves before 26 Jan. Furthermore, LcFT2 expression of those leaves was much higher than the other types of shoots, suggesting that mature leaves had higher expression levels of the floral promoter and the florigen gene. Also, trees with dark green terminal shoots had higher flowering rates, suggesting that the ability of the terminal shoots to produce flowers might be related to their high expression of $\mathrm{LcCO}$ and LcFT2.

In contrast to $C O$ and $F T, F L C$, a MADS domain-containing transcription factor is believed to act as a floral repressor (Michaels and Amasino, 1999). FLC was found to be expressed at the highest levels in the shoot apex where it represses expression of SUPPRESSOR OF OVEREXPRESSION OF CONSTANS 1 (SOC1) in Arabidopsis (Michaels, 2009; Michaels and Amasino, 2000). It is also expressed to a lesser extent in leaves where it acts to repress $F T$. In our study, we found that $L c F L C$ expression of the yellowish red 
leaves, with which branches had lower floral production, increased from $16 \mathrm{Jan}$. However, that of the dark green leaves, with which branches had higher floral production, declined to a level lower than the yellowish red leaves on 4 Feb., indicating that the dark green leaves had a decreasing expression of the floral repressor. It was suggested that high flowering rate of the dark green branches might be correlative with the decreased expression of $L c F L C$.

In conclusion, the results of this study provided physiological and molecular evidence to signify the importance of the maturity of terminal shoots (potential flowering branches) before floral induction for successful flowering. In litchi production, leaves of the terminal shoots should be dark green during floral induction and differentiation stages, and winter flushes should be removed or killed.

\section{Literature Cited}

An, H.L., C. Roussot, P. Suarez-Lopez, L. Corbesler, C. Vincent, M. Pineiro, S. Hepworth, A. Mouradov, S. Justin, C. Turnbull, and G. Coupland. 2004. CONSTANS acts in the phloem to regulate a systemic signal that induces photoperiodic flowering of Arabidopsis. Development 131:3615-3626.

Chaikiattiyos, S., C.M. Menzel, and S.T. Rasmussen. 1994. Floral induction in tropical fruit trees: Effects of temperature and water supply. J. Hort. Sci. 69:397-415.

Chen, H.B. and H.B. Huang. 2005. Low temperature requirements for floral induction in lychee. Acta Hort. 665:195-202.

Chen, H.B., H.B. Huang, and Z.L. Liu. 2004. Flower formation and patterns of carbohydrate distribution in litchi trees. Acta Hortic. Sin. 31:1-6 [in Chinese].

Coruzzi, G. and L. Zhou. 2001. Carbon and nitrogen sensing and signaling in plants: Emerging 'matrix effects'. Curr. Opin. Plant Sci. 4:247-253.

Davenport, T.L. and R.A. Stern. 2005. Flowering, p. 87-105. In: Menzel, C.M. and G.K. Waite (eds.). Litchi and longan. CABI, UK.

Gibson, S. 2005. Control of plant development and gene expression by sugar sensing. Curr. Opin. Plant Biol. 8:93-102.

Hayama, R. and G. Coupland. 2003. Shedding light on the circadian clock and the photoperiodic control of flowering. Curr. Opin. Plant Biol. 6:13-19.

Ito, A., H. Hayama, and Y. Kashimura. 2002. Sugar metabolism in buds during flower bud formation: A comparison of two Japanese pear [Pyrus pyrifolia (Burm.) Nak.] cultivars possessing different flowering habits. Sci. Hort. 96:163-175.

Jang, J.C. and J. Sheen. 1997. Sugar sensing in higher plants. Trends Plant Sci. 2:208-214.

Li, W.M., Y. Tao, Y.X. Yao, Y.J. Hao, and C.X. You. 2010. Ectopic over-expression of two apple Flowering Locus T homologues, MdFTI and $M d F T 2$, reduces juvenile phase in Arabidopsis. Biol. Plant. 54:639-646.

Liu, W.W., H.J. Kim, H.B. Chen, X.Y. Lu, and B.Y. Zhou. 2013. Identification of MVgenerated ROS responsive EST clones in floral buds of Litchi chinensis Sonn. Plant Cell Rep. 32:1361-1372.

Livak, K. and T. Schmittgen. 2001. Analysis of relative gene expression data using real-time quantitative PCR and the $2^{-\Delta \Delta C T}$ method. Methods 25:402-408.

Menzel, C.M., T.S. Rasmussen, and D.R. Simpson. 1995. Carbohydrate reserves in lychee trees (Litchi chinensis Sonn.). J. Hort. Sci. 70:245255.

Menzel, C.M. and D.X. Simpson. 1988. Effect of temperature on growth and flowering of litchi (Litchi chinensis Sonn.) cultivars. J. Hort. Sci. 63:349-360.

Michaels, S.D. 2009. Flowering time regulation produces much fruit. Plant Biol. 12:75-80.

Michaels, S.D. and R.M. Amasino. 1999. FLOWERING LOCUS C encodes a novel MADS domain protein that acts as a repressor of flowering. Plant Cell 11:949-956.

Michaels, S.D. and R.M. Amasino. 2000. Memories of winter: Vernalization and the competence to flower. Plant Cell Environ. 23:1145-1154.

Mouradov, A., F. Cremer, and G. Coupland. 2002. Control of flowering time: Interacting pathways as a basis for diversity. Plant Cell 14:S111-S130.

Samach, A., O. Hitoshi, E.G. Scott, S.D. Gary, S.S Zsuzsanna, F.Y. Martin, and C. George. 2000. Distinct roles of CONSTANS target genes in reproductive development of Arabidopsis. Science 288:1613-1616.

Sheen, J., L. Zhou, and J.C. Jang. 1999. Sugars as signaling molecules. Curr. Opin. Plant Biol. 2:410-418.

Wagner, D., R.W.M. Sablowski, and E.M. Meyerowitz. 1999. Transcription activation of APETALA1 by LEAFY. Science 285:582584.

Wang, Q.B., M.J. Chen, and Y.C. Li. 2004. Nondestructive and rapid estimation of leaf chlorophyll and nitrogen status of peace lily using a chlorophyll meter. J. Plant Nutr. 27:557-569.

Wellmer, F. and J.L. Riechmann. 2010. Gene networks controlling the initiation of flower development. Trends Genet. 26:519-527.

$\mathrm{Xu}$, C.J., W.J. Chen, K.S. Chen, and S.L. Zhang. 1998. A simple method for determining the content of starch-iodine colorimetry. Biotechnology. 8:41-43 [in Chinese].

Xue, Y.L. 1985. Plant physiology manual. Shanghai Science and Technology Publication, Shanghai, China.

Yamamoto, A., T. Nakamura, J.J. Adu-Gyamfi, and M. Saigusa. 2002. Relationship between chlorophyll content in leaves of sorghum and pigeonpea determined by extraction method and by chlorophyll meter (SPAD-502). J. Plant Nutr. 25:2295-2301.

Yang, Y.J., J. Klejnot, X.H. Yu, X.M. Liu, and C.T. Lin. 2007. Florigen (II): It is a mobile protein. J. Integr. Plant Biol. 49:1665-1669.

Yin, J.H., J.M. Lu, X.M. Huang, S. Luo, and Y.C. Lai. 2008. A study of changes in chlorophyll content and structure of leaf of Nuomici litchi cultivar during flower induction. J. Fruit Sci. 25:258-261 [in Chinese].

Zhou, B., H. Chen, X. Huang, G. Wu, Z. Hu, and Z. Gao. 2010a. Changes of antioxidant enzyme activity and hydrogen peroxide concentration in Litchi chinensis during floral differentiation. Acta Hort. 863:453-460.

Zhou, L., Y.T. Zhou, M.L. Wang, H.Y. Wang, and Y. Zhao. 2010b. Expressions and dimerization affinities of three highly identical APETALA3 genes in Brassica napus. Biol. Plant. 54:3340.

Zhou, B., N. Li, Z. Zhang, X. Huang, H. Chen, Z. Hu, X. Pang, W. Liu, and Y. Lu. 2012. Hydrogen peroxide and nitric oxide promote reproductive growth in Litchi chinensis. Biol. Plant. 56:321-329.

Zhou, B.Y. 2004. Responses of Stylosanthes guianensis to chilling and its regulation by abscisic acid. PhD diss., South China Agricultural University, Guangzhou, China [in Chinese].

Zhou, B.Y., H.B. Chen, and C.B. Wu. 2014. An overview on natural triggers and stress signals in relation to flowering in Litchi chinensis and Dimocarpus longan. Acta Hort. 1029:137144. 